\title{
Multi-Robot Exploration and Rendezvous on Graphs
}

\author{
Malika Meghjani and Gregory Dudek
}

\begin{abstract}
We address the problem of arranging a meeting (or rendezvous) between two or more robots in an unknown bounded topological environment, starting at unknown locations, without any communication. The goal is to rendezvous in minimum time such that the robots can share resources for performing any global task. We specifically consider a global exploration task executed by two or more robots. Each robot explores the environment simultaneously, for a specified time, then selects potential rendezvous locations, where it expects to find other robots, and visits them. We propose a ranking criterion for selecting the order in which potential rendezvous locations will be visited. This ranking criterion associates a cost for visiting a rendezvous location and gives an expected reward of finding other agents. We evaluate the time taken to rendezvous by varying a set of conditions including: world size, number of robots, starting location of each robot and the presence of sensor noise. We present simulation results to quantify the effect of the aforementioned factors on the rendezvous time.
\end{abstract}

Keywords - exploration, rendezvous, multi-robot coordination, random environments, sensor noise.

\section{INTRODUCTION}

This paper addresses algorithmic improvements to strategies for multi-robot rendezvous, and looks at extending new and existing strategies to larger number of robots than previously considered in [1]. Rendezvous is the problem of getting two or more agents to find and meet each other in (possibly unknown) environment. It is a problem class faced every day by biological organisms looking for a mate, people catching the bus, and perhaps even undercover secret agents.

In our work, we consider rendezvous in a topological environment in a worst-case scenario, where no communication is possible between the agents until they physically come together. This can arise in practice if the environment is very large relative to the communication bound, if a large number of communication channels are available or if there are reasons for the team to maintain radio silence.

We specifically analyze the rendezvous problem in the context of multi-robot exploration. The multi-robot exploration task requires mobile robots to uniquely cover a given region of interest and also meet as quickly as possible to share their respective information. This process involves continuous communication and planning between the agents for coordination [2]. In contrast, we consider the case where the robots do not communicate during exploration and attempt to meet at regular intervals to find and exchange information with each other. The meeting attempts are made

The authors are with the Centre for Intelligent Machines, McGill University, Montréal, Québec, Canada.

\{malika, dudek\}@cim.mcgill.ca by visiting potential rendezvous locations which are selected during exploration. These locations are visited based on a rendezvous strategy and a ranking criterion. The rendezvous strategy gives the probability of visiting a potential rendezvous location and the ranking criterion provides an ordering relation for visiting them. In this work, we assume that potential rendezvous locations can be selected based on some sensor property which we refer to as distinctiveness.

The main contributions in this paper are: combining exploration and rendezvous, evaluating the effect of initial distance between the mobile agents on the time to explore and rendezvous, analyzing the effect of noisy sensors on the rendezvous time and proposing generalized algorithms which are applicable to any number of agents. In addition, we postulate a hybrid ranking criterion based on our empirical observations. The following sections give an overview of: related work for multi-robot rendezvous problem in realistic and graph-like environments in Section II, our proposed approach is described in Section III along with a summary of our algorithms and the simulation results are presented in Section IV.

\section{RELATED WORK}

The rendezvous problem was first introduced as a search problem in game theory and is known for its use in the mobile hider game, princess and monster [3]. Since then, it has been extensively studied based on two world models: graphs and simple geometric figures (such as lines, circles and polygons) [4]. A survey of results for mobile agent rendezvous is given in [5]. The theoretical results from these abstract world models have been extended to real-world environments with modifications to the rendezvous definition itself. We focus on the work where rendezvous is applied to the global task of exploration.

The task of scheduling a meeting between robots that are in constant communication has been addressed by several authors [6], [7], [8] in context of multi-robot exploration. This problem is quite different from our present work since communication makes many synchronization issues straightforward. Also, the distance constraints on communication range requires the agents to be in the vicinity of each other which makes exploration less efficient. An alternative to overcome this drawback is to divide the multi-robot team into small groups and start exploration from different locations. These small groups communicate via a central agent to share maps and distribute their work. This specific approach was proposed in [9]. Another hierarchical approach was introduced in [10] which considers the problem of rendezvous after momentarily splitting up. Specifically, the robots in the 
team are assigned roles for exploring and relaying information. The explorer robots repeatedly split from the relay robots for exploration. The relay robots share information with a central agent and try to rendezvous with the explorer by estimating its current location using topological cues. This short-range rendezvous problem without any communication is similar to our present work with the difference being that in our work, the agents begin exploration from unknown locations and they do not have an estimate of the locations of other agents.

The variant of the rendezvous problem where the robots initially start exploring from different unknown locations and communicate only by physically meeting each other is rarely addressed in robotics applications, yet it occurs often between biological agents. One particular work which considers this problem is given in [11]. Our work is motivated by their rendezvous strategies which are extended for more than two robots. In addition, we also introduce a cost efficient ranking criteria for combining exploration with rendezvous.

This paper only deals with the problem of rendezvous in abstract graph worlds. However, the same rendezvous strategies and ranking criteria can be applied to a real world environment by extracting a graph from the explored map. This problem in particular, is addressed in our previous work [1] where we consider the practical issues such as: mobile robot navigation, mapping, graph extraction and measuring distinctiveness of the world structure for obtaining potential rendezvous locations.

\section{Proposed Approach}

Our goal is to combine exploration and rendezvous for multiple robots in abstract simulated environments of varying sizes. The robots are initially distributed in the environment at random locations restricted by initial distance criterion to avoid overlap of the explored regions. The robots then begin exploration from these locations and make a rendezvous attempt at specified intervals. The details of random environment generation, exploration along with initial distance criterion and rendezvous are given in the following sections.

\section{A. Random Environment Generation}

Since experimental results on ensembles of graphs can be dependent on the graph family, our experimental data is based on two distinctly different families of planar graphs as shown in Fig. 1. They are:

- Grid-based world

- Triangulation-based world

The triangulation-based world is constructed by starting with triangulation of a set of random points and removing random edges while preserving the connectivity of the graph. In particular, a set of $N$ random points are generated ensuring that no two points are closer than a minimum distance. This set of random points is used as input to a delaunay triangulation [12] algorithm, the resulting triangulation is interpreted as a graph where the random points (triangle vertices) are the nodes of the graph and the triangle sides are used as the graph edges. Each graph edge is assigned

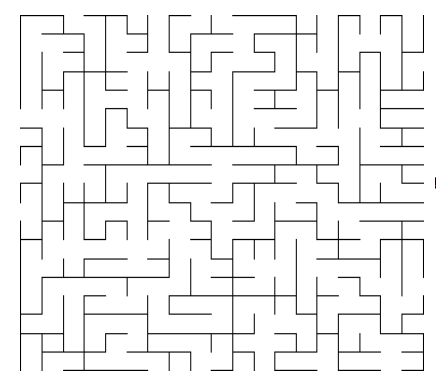

(a) Grid-based world

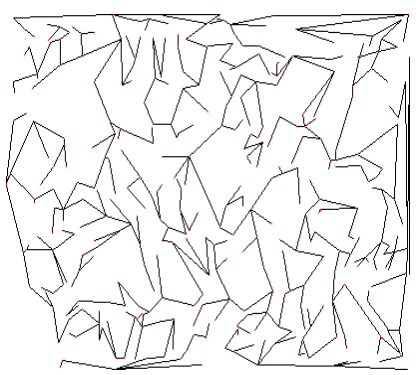

(b) Triangulation-based world
Fig. 1. Random Environments

a random weight, and a minimum spanning tree (MST) is calculated based on these weights. The edges are then removed repeatedly from the graph as long as they are not a part of the MST, thus ensuring both the connectivity and the randomness of the resulting structure as shown in Fig. 1(b).

The same procedure is used for grid-based world generation with only difference that we initially begin with a complete grid structure for a given number of nodes which are separated by a fixed edge length. These edges are then removed iteratively while satisfying connectivity and randomness in the graph, as described above. The random grid thus obtained, models a complete road network with some fractions of the roads missing, as has been previously referred to "Montreal graph" [13]. An example of a gridbased world with 400 nodes is presented in Fig. 1(a).

\section{B. Rendezvous}

The primary challenge in a multi-robot rendezvous problem is to define the rendezvous process itself. In our current work, a successful rendezvous is defined as the state when a transitive rendezvous relation is established between the agents. Consider three robots $r_{i}, r_{j}$ and $r_{k}$, if $r_{i}$ has met $r_{j}$ at time $T_{1}$ and $r_{j}$ has met $r_{k}$ at time $T_{2}$ then all the information collected by agent $r_{j}$ from agent $r_{i}$ is shared with $r_{k}$ at time $T_{2}$ thus constituting the rendezvous transitive property. In addition, we assume that the agent $r_{j}$ can coordinate with agent $r_{i}$ to share any new information received from agent $r_{k}$, after its first successful rendezvous. Therefore, based on the aforementioned property we consider all the robots to have a successful rendezvous at time $T_{2}$.

There are two possible ways the robots can coordinate with each other after a successful rendezvous: (a) either they can move together as a pack or (b) they can disperse in unexplored regions and meet at a known location based on a scheduling process similar to task partitioning. These cases are beyond the scope of our current subject of interest and we do not consider them in our present work.

In order to keep track of multi-robot rendezvous at different intervals we define a rendezvous matrix, $\Gamma$ which is similar to an adjacency matrix in graph theory. It is a symmetric matrix of size $n \times n$ where $n$ is the number of robots. The rows and columns of the matrix correspond to the rendezvous outcome between the agents. For example, as shown in the matrix $\Gamma$, the row $r_{i}$ represents the rendezvous 
outcome of agent $r_{i}$ with respect to the agents $r_{1}$ through $r_{n}$. The elements of this matrix are binary with value 1 representing a successful rendezvous and value 0 representing no rendezvous. The rendezvous transitivity property is then evaluated to be true if $\Gamma^{n}$ is equal to a non-zero matrix. A formal multi-robot rendezvous process is presented in Algorithm 1.

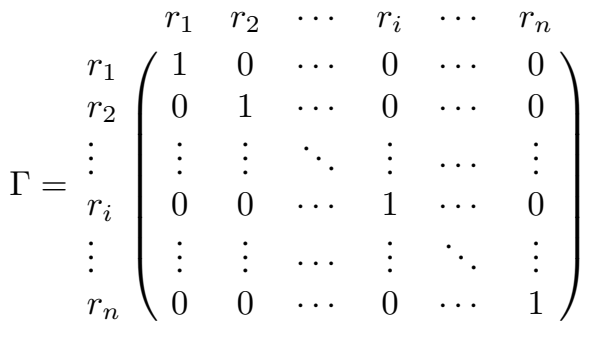

- At the rendezvous time $T_{t}$ the robots attempt to meet.

- Each robot $r_{i}$ selects a location $p_{k}^{r_{i}}$ to visit on this attempt, thus defining a sequence of attempted rendezvous locations $p_{k}^{r_{i t}}$.

- Each robot travels to its appointed location $p_{k}^{r_{i t}}$ and, if $p_{k}^{r_{i t}}=p_{k}^{r_{j t}}$, that is, robots $r_{i}$ and $r_{j}$ are in the same place then we mark the entry $\left(r_{i}, r_{j}\right)$ and $\left(r_{j}, r_{i}\right)$ in $\Gamma$ matrix as 1.

- If the elements of the matrix $\Gamma^{n}$ are all of non-zero value then the multi-robot rendezvous process is considered to be successful.

- In the event of a failed rendezvous, the robots continue their background activity until the next rendezvous interval.

Algorithm 1: Multi-robot rendezvous process

As mentioned in Section I, we implement three rendezvous strategies for more than two robots, namely asymmetric, symmetric and exponential. The first two strategies correspond to robot roles during the rendezvous period. In the asymmetric strategy, one of the robots is stationary at a node while the other robots are allowed to move. These roles are pre-determined before the start of exploration or rendezvous processes. In the symmetric strategy all robots move and hence do not require any pre-arranged role assignments. In the exponential strategy, robots select possible rendezvous locations at random, weighted by an exponentially decreasing function of distinctiveness.

The rendezvous strategies are combined with ranking criteria that provide an order for visiting the potential rendezvous locations. We propose three ranking criteria which are based on the distinctiveness measure of the location, linear-distance and sigmoid-distance from current location to the potential rendezvous point. These rendezvous strategies and ranking criteria are derived from our previous work [1].

The distinctiveness measure considers the uniqueness of the potential rendezvous locations for ranking. This measure is a function of individual robot sensors and may vary due to sensor noise. Examples of distinctiveness measure for indoor environment are: hallway intersections or entrances and exits of buildings. In our model, we simulate the effect of natural variability of the environment, by using random values in the range $[1, N]$ for distinctiveness $D\left(p_{k}\right)$ of any location $p_{k}$.

The distinctiveness-based ranking does not account for the amount of work required to visit a rendezvous location. This implies that in case of a failed rendezvous attempt at far locations the robot will lose substantial resources. Hence, we propose a cost-reward model which accounts for the cost of making a rendezvous attempt against the reward of the uniqueness of the location. The cost is measured in terms of the node-to-node distance $d\left(c_{k}, p_{k}\right)$ between the robot's current location $c_{k}$ and the potential rendezvous point $p_{k}$. We consider two variants of the cost function: lineardistance and sigmoid-distance. The linear-distance weighted distinctiveness, $D_{L}\left(p_{k}\right)$ is given in Eq. 1.

$$
D_{L}\left(p_{k}\right)=\frac{D\left(p_{k}\right)}{d\left(c_{k}, p_{k}\right)}
$$

The sigmoid-distance weighted distinctiveness, $D_{S}\left(p_{k}\right)$ is given in Eq. 2. This is obtained by applying a sigmoid function $S$ to the distance measure i.e. $S\left(d\left(c_{k}, p_{k}\right)\right)$. Intuitively, this ranking criterion gives larger weights to the closer rendezvous locations and smaller weights to the farther ones.

$$
D_{S}\left(p_{k}\right)=\frac{D\left(p_{k}\right)}{S\left(d\left(c_{k}, p_{k}\right)\right)}
$$

The pair-wise combination of the rendezvous strategies with ranking criteria provides nine variants of our algorithm. We test each of these variants with respect to the rendezvous time and infer the best combination in the ideal and noisy cases. These cases are discussed in the following section.

\section{Noisy Sensors}

The ideal-case for rendezvous, refers to the situation when the agents have synchronized clocks and noise-free sensors to meet precisely at desired time and location. In practical scenarios, the clocks of the agents may not synchronize which can cause variations in the start time for exploration and rendezvous. We deal with this situation in real environments, by allowing a wait time $\delta$ at each potential rendezvous location to compensate for small delays. The second condition for ideal-case which assumes noise-free sensors, can be easily violated in real environments when agents vary in their position while measuring the distinctiveness of a location. The distinctiveness measure is a function of the environment structure and agent's position, assuming noisefree sensors. We remove these dependencies in our analysis by using topological graph worlds and allowing rendezvous to occur only on the nodes of the graphs.

In the situation when the sensors are noisy, the agents may have a mismatch in the rank ordering of the rendezvous locations and hence the visiting preferences. This factor effects the rendezvous time for different ranking criteria which we quantify in our analysis. We model the sensor noise as a Gaussian function $G$ and simulate it by applying this function to the distinctiveness measure $D\left(p_{k}\right)$ of the potential 
rendezvous location $p_{k}$ as given in Eq. 3. The distance measure required for cost-reward model, is applied after considering the noise function. In our experiments presented in Section IV, we use $\sigma=3$, as the variance of the Gaussian function.

$$
N\left(p_{k}\right)=G\left(D\left(p_{k}\right), \sigma\right)
$$

\section{Exploration}

Multi-robot exploration requires the robots to be initially dispersed in the environment such that they uniquely cover the given region of interest. In our work, we deploy the robots based on an initial distance criterion according to which the distances between the agents are selected as greater than or equal to a fraction of the environment size. The agents are unaware of the initial separation distance between them and we use this criterion only to analyze its effect on different rendezvous strategies.

We implement this criterion by randomly selecting a location in the environment as the initial location of the first robot and obtaining a set of points on the circumference of a circle with radius equal to the required distance. A random location is then picked from these locations and it is considered as the initial location for the 2 nd robot. This process is repeated to obtain the initial locations for all the remaining robots. An example of the initial dispersion of the mobile agents on a grid-based world is shown in Fig. 2. It must be noted that the initial distance refers to the euclidean distance on the graph and does not require the node-to-node distance information for this calculation.

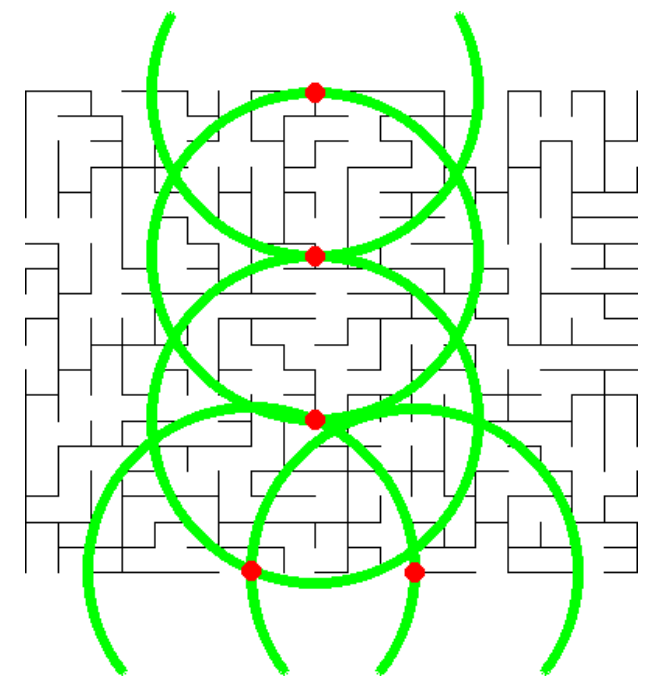

Fig. 2. Initial robot locations: The smaller circles indicate the locations of a particular robot and the rings around them represent the distance of separation between them

The mobile agents begin at these locations and explore the environment in bread-first order. The exploration is performed for a fixed period and is followed by rendezvous attempts within the explored regions of respective robots. The regions explored by individual agents may not overlap, resulting in unsuccessful rendezvous attempts. In this case, the exploration is resumed from the same frontier node where it was disrupted for rendezvous.

Our current implementation, allows rendezvous to occur only on the nodes of the graph and not on the edges. Hence, each graph node is considered as the potential rendezvous location. In addition, we assume the agents move only over the nodes without traversing the edges on the graph and thus we do not deal with navigational issues such as obstacle avoidance.

\section{EXPERIMENTAL RESULTS}

This section presents a statistical analysis of the results for multi-robot rendezvous algorithms. We specifically study the effect of sensor noise, initial-distance between the agents, environment size and number of robots, on the time taken to rendezvous. The time taken to rendezvous is measured in terms of the node-to-node distance for all the experiments. The results for each of these cases is discussed below in detail.

We first present a detailed comparison of three-robot rendezvous outcomes for the ideal and noisy cases (in Figs. 3, 4 and 5) using a grid-based environment with 400 nodes. These results are averaged over 9 different starting locations which are split as 3 locations for each of the 3 initial separation distances: small, medium and large (corresponding to 1/8, 1/4 and $1 / 2$ the factor of the given environment size). Results for larger number of robots follow essentially the same pattern and are summarized subsequently in Fig. 8.

Fig. 3 gives an overview of the rendezvous strategies and ranking criteria with respect to the total time taken to rendezvous. It can be observed that asymmetric rendezvous strategy, where one robot is stationary and all the other robots are moving, is the best strategy with minimum time to rendezvous in both ideal and noisy cases. In the ideal-case, the sigmoid-distance weighted distinctiveness ranking performs the best for all the rendezvous strategies which is followed by distinctiveness-only and linear-distance weighted distinctiveness ranking. The case with noise clearly suggests that purely distinctiveness based ranking performs worst when compared to the linear and sigmoid distance based ranking. These two trends seen in the ideal and noisy cases suggest that the costreward model has better performance when there are noisy sensors.

The results presented in the following sub-section are all averaged over the rendezvous strategies in order to compare the different ranking criteria. The variation in the total time taken to rendezvous with respect to the total number of combined rendezvous attempts made by all the robots is presented in Fig. 4. It can be observed in the ideal-case, that the number of attempts vary almost linearly with total time taken to rendezvous. However, this pattern changes in noisy case where the cost-reward model reduces the distance traveled per rendezvous attempt. The noisy case also suggest that linear-distance based ranking requires approximately the same number of rendezvous attempts as the other two ranking criteria but with much smaller distances. 


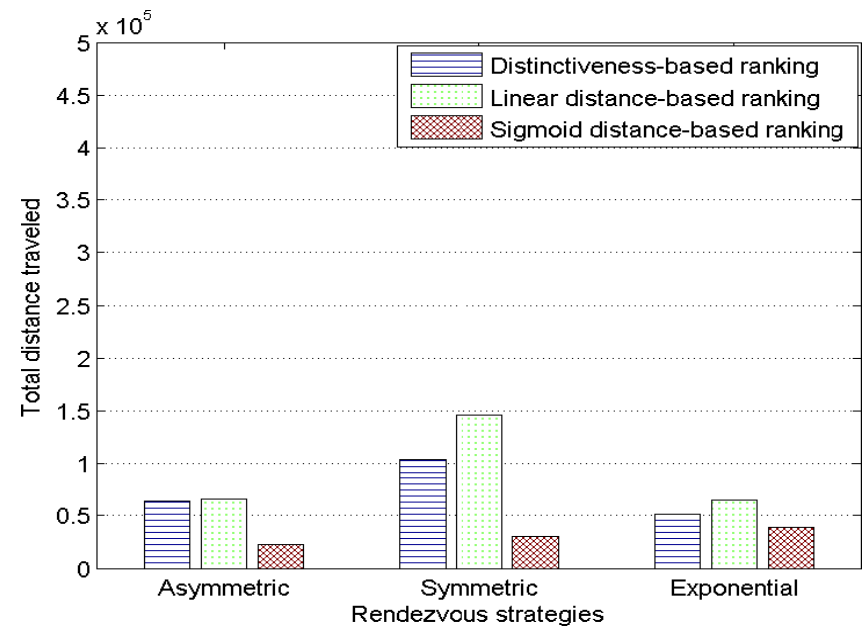

(a) Ideal

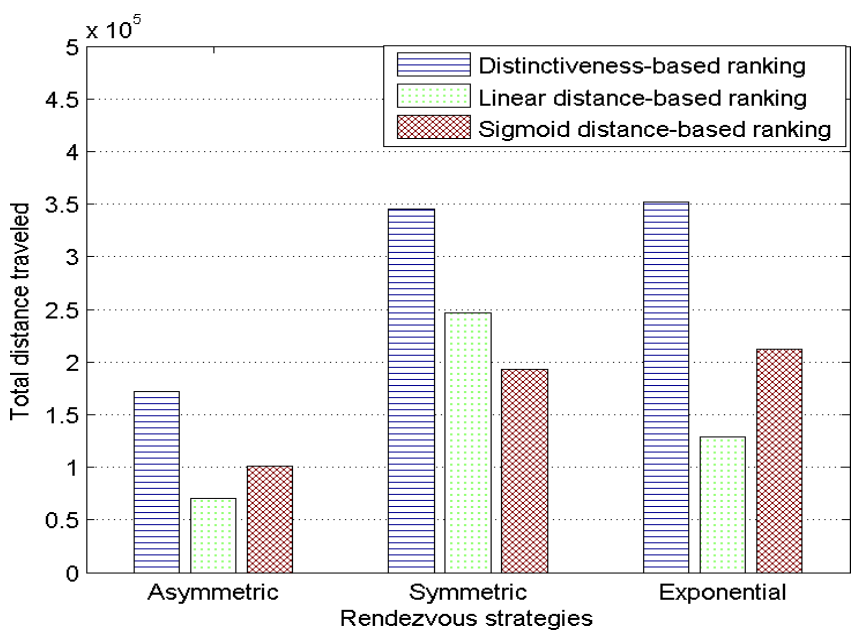

(b) Noisy

Fig. 3. Total distance traveled for different rendezvous strategies and ranking criteria

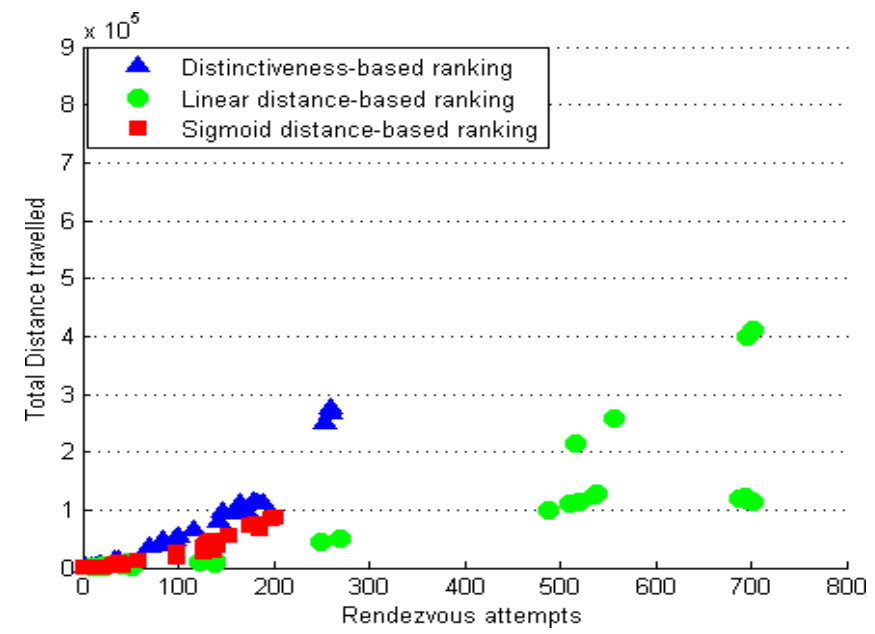

(a) Ideal

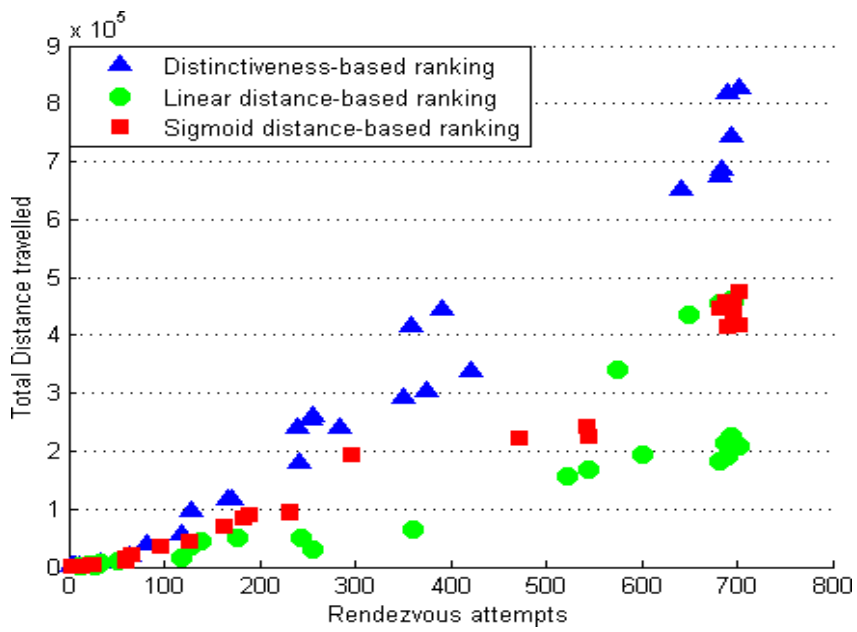

(b) Noisy

Fig. 4. Number of rendezvous attempts and distance traveled for different ranking criteria

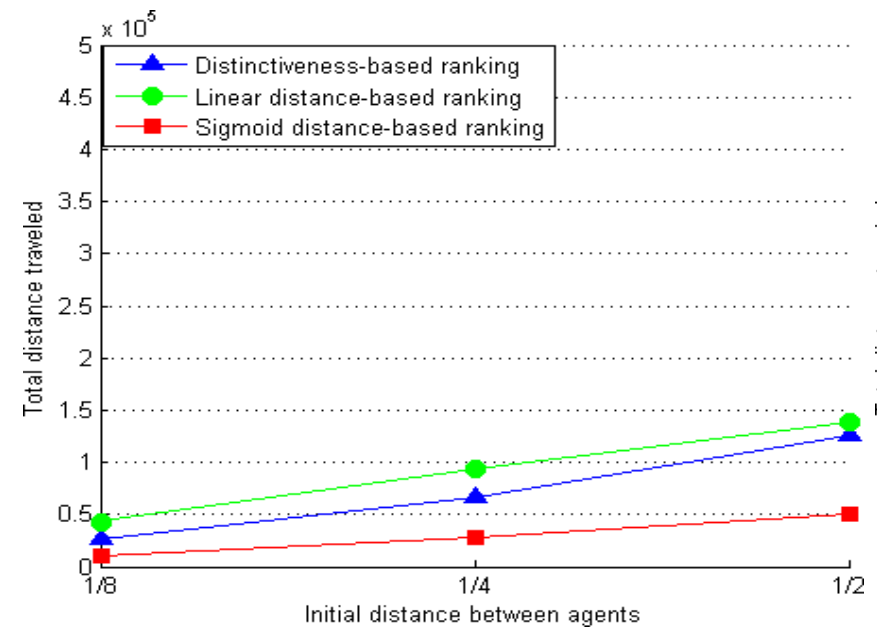

(a) Ideal

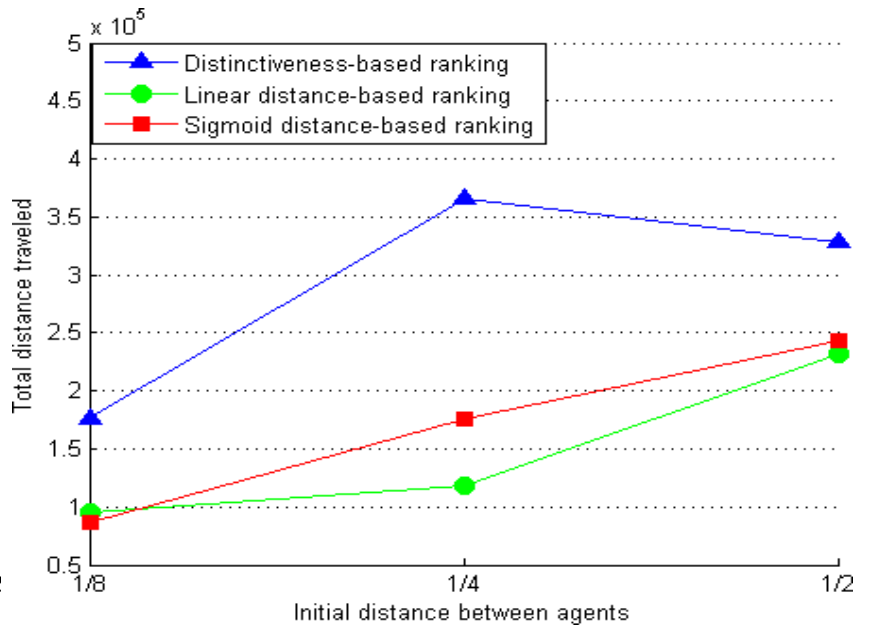

(b) Noisy

Fig. 5. Total distance traveled to rendezvous for different ranking criteria as a function of initial distance between the agents 
A comparison of the ranking criteria with respect to the initial distance is presented in Fig. 5. It can be observed that as the initial distance of separation increases, the rendezvous time also increases in both ideal and noisy cases. In the ideal-case with the large initial distance of separation (i.e. $(1 / 2)^{*}$ environment size), the sigmoid-based ranking criteria performs significantly better than the distinctiveness-based measure since it ranks the closer landmarks higher than the farther ones. However, in the noisy case the linear-distance based ranking takes over sigmoid-based ranking.

A summary of the results with different environment sizes and the time taken to rendezvous are presented in Fig. 6. In this plot, the environment size is measured in-terms of the number of nodes in the graph. These results were obtained for three robots in the ideal-case. It can be observed that the difference between the total distance traveled or time taken to rendezvous gradually becomes significant as the size of the environment increases. It can thus be concluded that as the environment size increases, the cost-reward model performs better when compared to the distinctiveness-based ranking.

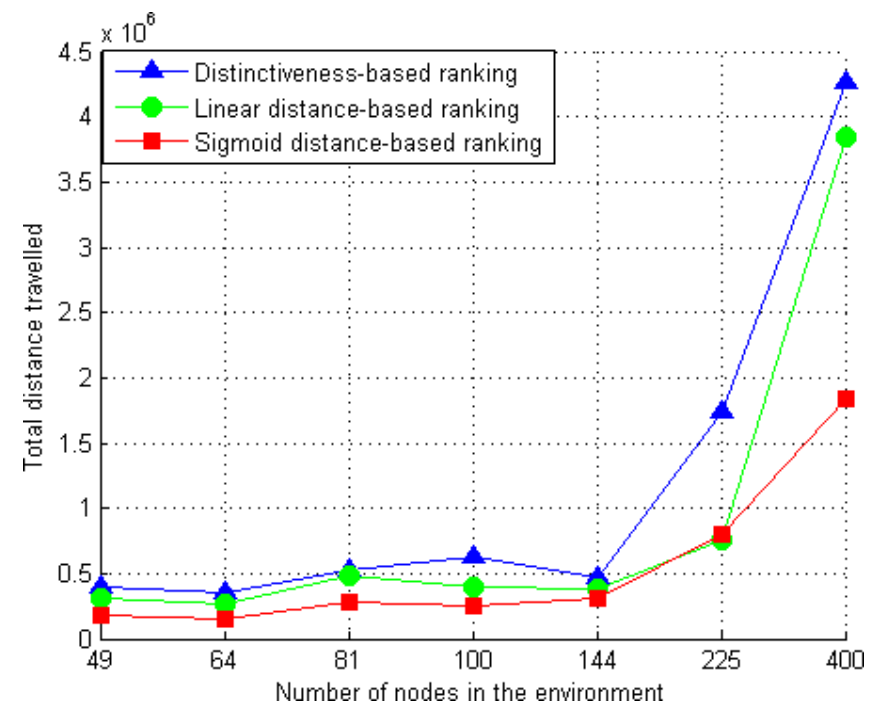

Fig. 6. Total distance traveled or the time taken to rendezvous as a function of environment size: Ideal case

The results discussed above clearly indicate the need of the cost-reward model to speed-up the rendezvous process. However, if the agents have noise-free sensors and have explored the complete graph then the cost-reward model is not useful since the agents can rendezvous at the most distinctive node in one attempt only. In order to choose between the different ranking criteria, we analyze the effect of variation in the initial number of common nodes on the total time to rendezvous. We allow the agents to only explore initially until a desired number of common nodes are achieved and then continue with combination of rendezvous and exploration attempts.

We measured this variation for different initial-distance of separation between three agents, in a grid-base environment with 400 nodes and ideal sensors. These results are presented in Fig. 7. When the environment is completely explored by all the agents, the distinctiveness based ranking criterion performs the best, as discussed above. However, when the number of common nodes decrease, the distinctiveness ranking is less effective and the sigmoid-based ranking shows superior performance. In our tests, this transition occurs when there is roughly $(5 / 8)^{t h}$ or less of the map is shared between the robots. Hence, for an effective combination of rendezvous and exploration, these results suggest an implementation of cost-reward model for rendezvous until 50\% of the environment is explored and then switch to distinctiveness based ranking in case of unsuccessful rendezvous attempts.

Finally, we present the result for multi-robot $(\geq 3)$ rendezvous in ideal-case, over a grid-based environment with 400 nodes in Fig. 8. This plot illustrates the variation in the time taken to rendezvous with respect to the number of robots. It can be observed that as the number of robots increase, the time taken for multi-robot rendezvous increases for all the ranking criteria. However, the cost-reward based ranking criteria (linear and sigmoid distance-based ranking) records shorter rendezvous time when compared to distinctiveness based ranking. The cost-reward model saves time by making rendezvous attempts at best nodes in the vicinity and if the attempt is unsuccessful it continues to explore. This results in a faster increase in the number of nodes explored and hence the probability of common nodes explored between the agents, when compared to distinctiveness-based measure.

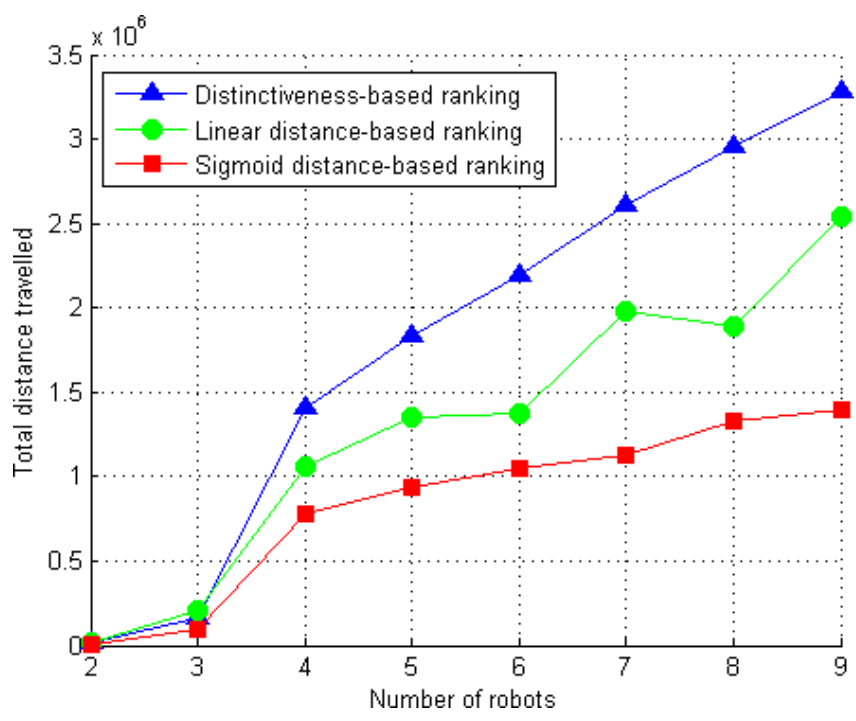

Fig. 8. Total distance traveled or the time taken to rendezvous as a function of number of robots: Ideal case

\section{Discussion AND CONCLUSION}

Our results from Section IV, show that the amount of information obtained from the environment has implications on how to perform rendezvous. Thus, we formulate a new ranking criterion which depends on the extent of exploration completed. We derive this criterion based on the results in Fig. 7. These results suggest that when half of the environment size is explored, there is a switch over of ranking preference from distinctiveness-based measure to cost-reward 


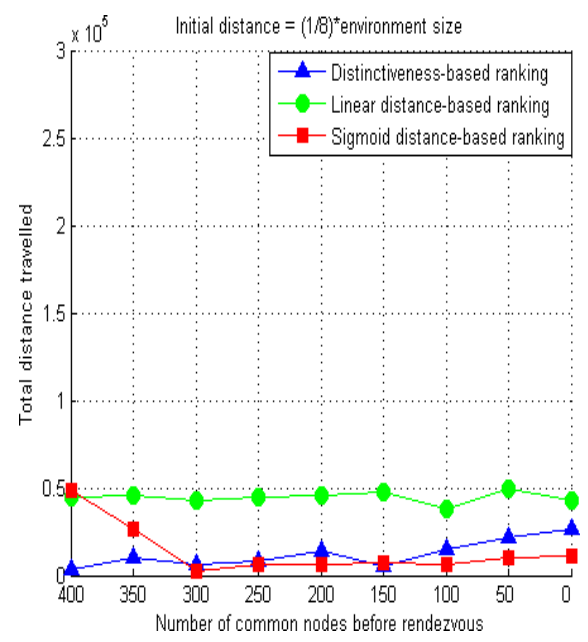

(a) Small initial distances

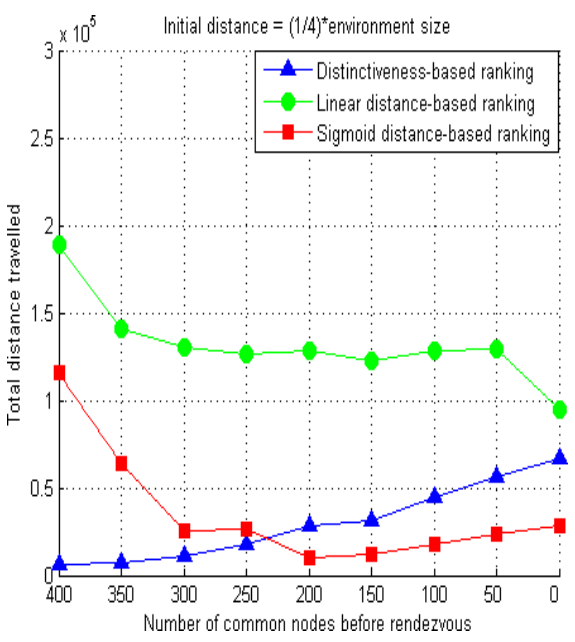

(b) Medium initial distances

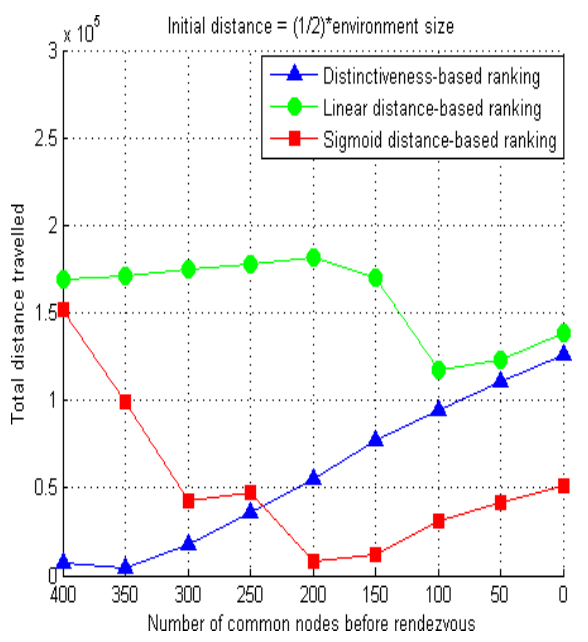

(c) Large initial distances

Fig. 7. Effect of variation in number of nodes overlapping on ranking criteria

model. Hence, we propose a combination of the two ranking criteria in Eq. 4, to obtain a hybrid ranking $D^{\prime}\left(p_{k}\right)$ for any potential rendezvous location $p_{k}$.

$$
D^{\prime}\left(p_{k}\right)=\alpha D\left(p_{k}\right)+(1-\alpha) D_{S}\left(p_{k}\right)
$$

The parameter $\alpha$ in the Eq. 4 is a continuous variable that can be used for switching between distinctiveness $D\left(p_{k}\right)$ and sigmoid-distance weighted distinctiveness $D_{S}\left(p_{k}\right)$ ranking methods and it can be obtained using the following equation:

$$
\alpha=\left(1-e^{-(2 n / N)}\right)
$$

The variables $n$ and $N$ represent the current number of nodes explored and estimated total number of nodes in a bounded environment, respectively. Hence, when $n$ is small implying there are not many nodes explored, the probability of number of overlapping nodes between the agents is low which provides a small value of $\alpha$ and results in a larger weight to the sigmoid-distance weighted distinctive ranking $D_{S}\left(p_{k}\right)$. In contrast, when $n$ is large the $\alpha$ value is large and the hybrid ranking criterion $D^{\prime}\left(p_{k}\right)$, biases towards the distinctiveness measure $D\left(p_{k}\right)$.

In conclusion, we addressed the problem of combining multi-robot exploration and rendezvous tasks in random topological environments. The abstraction of world structure and distinctiveness measure in these world environments made it easier to analyze the joint problem. The cost-reward model was shown to be a better ranking criterion for both ideal and noisy cases. The evaluation of the rendezvous process with noisy sensors provided a practical platform for testing our algorithms. In addition, our proposed algorithms were shown to be easily generalizable for any number of robots and can be applied in any environment with the framework given in [1]. These aspects of our work combined together makes it a novel contribution to multi-robot rendezvous.

The future direction for our work is to apply it on real robots to perform multi-robot rendezvous in radio-silent environments. We also intend to obtain the theoretical bounds on our algorithm to compare them against our simulated results.

\section{ACKNOWLEDGEMENT}

The authors would like to acknowledge Dr. Ioannis Rekleitis for his triangulation-based world generation code.

\section{REFERENCES}

[1] M. Meghjani and G. Dudek. Combining multi-robot exploration and rendezvous. In CRV '11: Proceedings of the 2011 Canadian Conference on Computer and Robot Vision, pages 80-85. IEEE Computer Society, May 2011.

[2] I. Rekleitis, A.P. New, E.S. Rankin, and H. Choset. Efficient boustrophedon multi-robot coverage: an algorithmic approach. Annals of Mathematics and Artificial Intelligence, 52(2):109-142, 2008.

[3] S. Alpern and S. Gal. The theory of search games and rendezvous. Springer, 2003.

[4] A. Dessmark, P. Fraigniaud, and A. Pelc. Deterministic rendezvous in graphs. Algorithms-ESA 2003, pages 184-195, 2003.

[5] E. Kranakis, D. Krizanc, and S. Rajsbaum. Mobile agent rendezvous: A survey. Structural Information and Communication Complexity, pages 1-9, 2006.

[6] I. Rekleitis, G. Dudek, and E. Milios. Multi-robot collaboration for robust exploration. In Proceedings of International Conference in Robotics and Automation, pages 3164-3169, San Francisco, USA, April 2000.

[7] W. Burgard, M. Moors, D. Fox, R. Simmons, and S. Thrun. Collaborative multi-robot exploration. In IEEE International Conference on Robotics and Automation, volume 1, pages 476-481, 2000.

[8] N. Atay and B. Bayazit. Emergent task allocation for mobile robots. In Proceedings of Robotics: Science and Systems, 2007.

[9] R. Vincent, D. Fox, J. Ko, K. Konolige, B. Limketkai, B. Morisset, C. Ortiz, D. Schulz, and B. Stewart. Distributed multirobot exploration, mapping, and task allocation. Annals of Mathematics and Artificial Intelligence, 52(2):229-255, 2008.

[10] J. de Hoog, S. Cameron, and A. Visser. Selection of rendezvous points for multi-robot exploration in dynamic environments. In International Conference on Autonomous Agents and Multi-Agent Systems, 2010.

[11] N. Roy and G. Dudek. Collaborative exploration and rendezvous: algorithms, performance bounds and observations. Autonomous Robots, 11(2):117-136, Sept. 2001.

[12] B. Delaunay. Sur la sphère vide [on the empty area]. Izvestia Akademii Nauk SSSR, Otdelenie Matematicheskikh i Estestvennykh Nauk, 7:793800, 1934.

[13] G. Dudek, M. Jenkin, E. Milios, and D. Wilkes. Map validation and robot self-location in a graph-like world. Robotics and autonomous systems, 22(2):159-178, 1997. 